\title{
Effect of Dissipative and Dispersive DNG Material Coating on the Scattering Behavior of Parallel Nihility Circular Cylinders
}

\author{
Shakeel Ahmed, ${ }^{1}$ Muhammad Naveed, ${ }^{2}$ Abdul Ghaffar, ${ }^{3}$ \\ Aqeel A. Syed, ${ }^{1}$ and Q. A. Naqvi ${ }^{1}$ \\ ${ }^{1}$ Department of Electronics, Quaid-i-Azam University, Islamabad 45320, Pakistan \\ ${ }^{2}$ Department of Physics, National University of Sciences and Technology (NUST), \\ Islamabad 44000, Pakistan \\ ${ }^{3}$ Department of Physics, University of Agriculture, Faisalabad, Pakistan \\ Correspondence should be addressed to Q. A. Naqvi, nqaisar@yahoo.com
}

Received 12 November 2010; Revised 29 January 2011; Accepted 16 March 2011

Academic Editor: J. Jiang

Copyright (C) 2011 Shakeel Ahmed et al. This is an open access article distributed under the Creative Commons Attribution License, which permits unrestricted use, distribution, and reproduction in any medium, provided the original work is properly cited.

\begin{abstract}
Electromagnetic scattering from coated nihility circular cylinders, illuminated by E-polarized plane wave, is investigated using an iterative procedure. Cylinders are infinite in length. The boundary conditions are applied on the surface of each cylinder in an iterative procedure in order to solve for the field expansion coefficients. The effect of different types of the coating layers including double positive (DPS) and double negative (DNG) on the alteration of the forward and backward scattering has been observed. Specially, the effect of dispersive and dissipative DNG coating layer has been focused. Numerical verifications are presented to prove the validity of this formulation by comparison with the published literature.
\end{abstract}

\section{Introduction}

Scattering from multiple cylinders in free space has been studied by many researchers [114]. For the analysis of multiple cylinders, different techniques had been used to analyze the scattering between nearby conducting circular cylinders [1]. Twersky [2] extended the multiple scattering problem to an $N$ number of cylinders for the first time. He expressed the total radiation field as an incident field plus a scattered field of various orders. Burke et al. [3] derived the multiple scattering solution for $N$ cylinders as a series of Hankel functions. Ragheb and Hamid [4] studied the scattering of plane waves by $N$ circular cylinders. They used conducting circular cylinders to simulate a cylindrical reflector [5]. 
Furthermore, a rigorous solution had been introduced to solve the scattering problem from an array of dielectric or conductor cylinders for a plane wave excitation of normal incidence [6]. Elsherbeni and Kishk [7] used the principle of equal volume model to study any twodimensional cylindrical object of arbitrary cross section by an array of circular cylinders. Elsherbeni et al. [8] presented an iterative scattering of a Gaussian beam by an array of circular conducting and dielectric cylinders. Elsherbeni gave a comparative study of twodimensional multiple scattering techniques [9]. Yin et al. [10, 11] studied the scattering of electromagnetic waves by an array of circular bianisotropic cylinders in the case of normal incidence. The scattering of an obliquely incident plane wave on an array of parallel dielectric circular cylinders of arbitrary radii and positions was considered by Henin et al. [12]. Ahmed and Naqvi have studied the scattering of electromagnetic waves from parallel perfect electromagnetic conductor (PEMC) cylinders [13], strip and strip grating simulated by circular PEMC cylinders [14]. Roumeliotis and Ziotopoulos studied the scattering by a circular cylinder parallel with another one of small radius [15].

Concept of nihility medium has been introduced by Lakhtakia $[16,17]$ and has attracted the attention of many researchers [18-22]. Nihility medium is a medium in which both relative permittivity and permeability are null valued [16, 17]. Due to this fact, the medium does not allow the electromagnetic energy to propagate in it $[16,17]$ because

$$
\begin{aligned}
& \nabla \times E=0, \\
& \nabla \times H=0 .
\end{aligned}
$$

Knowing the fact that nihility medium is unachievable but having the concept of its simulation in some narrow frequency range, it still attracts scientists [23, 24].

Electromagnetic scattering by coated circular cylinders is a classical problem in electromagnetics and was investigated by several researchers [25-29]. Shen and Li [25] studied electromagnetic scattering by a conducting cylinder coated with materials having negative permittivity and permeability also known as double-negative (DNG) material. The scattered field for two plane waves incident on a circular cylinder covered by a dielectric material was investigated by Mushref [26]. A closed series solution of electromagnetic scattering by an eccentric coated cylinder in matrix form was achieved by Mushref [27], while Sun et al. [28] analyzed the electromagnetic radiation of a line source scattered by an infinite conducting cylinder coated with a left-handed material (LHM material). Irci and Ertürk [29] used the DNG and single-negative (SNG) coated conducting cylinder to achieve transparency and scattering maximization.

In this paper, an iterative solution for the scattered field from $M$ parallel coated nihility circular cylinders is derived. The cylinders are excited by $E$-polarized plane wave. In response to this field each of the coated cylinders scatters this field which is referred to as the initial or zeroth-order scattered field of each cylinder. Then, the zeroth-order scattered field from M-1 coated cylinders is considered as the incident field on the remaining cylinders. Thus, a first-order scattered field is generated. This process can be repeated for each coated cylinder. In order of the interaction between the cylinders, the appropriate boundary conditions are satisfied on the surface of the coating layer as well as on the surface of core cylinder.

\section{Formulation}

Consider a number of parallel circular nihility cylinders excited by an incident plane wave as shown in Figure 1. They are supposed to be numbered from 1 to $M$, while each is 


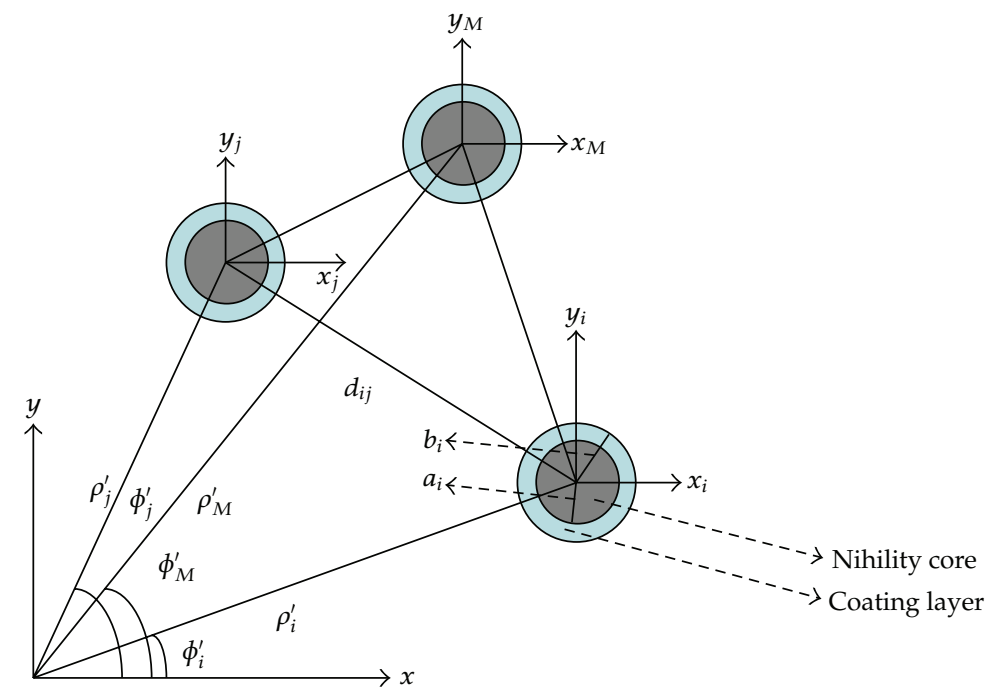

Figure 1: Geometry of coated nihility circular cylinders.
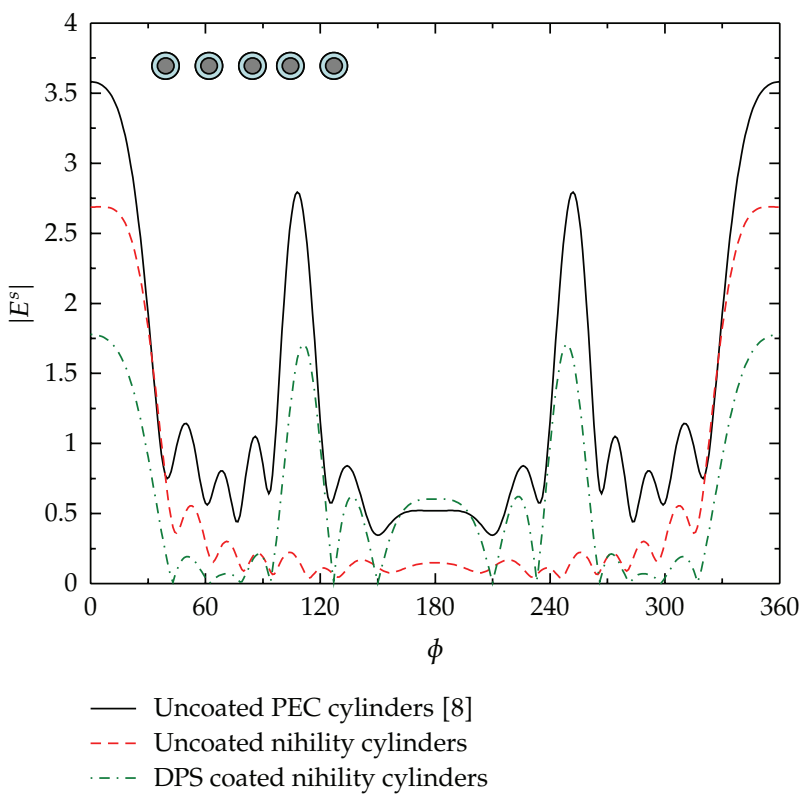

Figure 2: Far-zone scattered field pattern due to DPS $\left(\varepsilon_{1}=5, \mu_{1}=1\right)$ coated nihility circular cylinders (horizontal configuration) $a=0.1 \lambda_{0}, b=0.12 \lambda_{0}$, and $d=0.75 \lambda_{0}$.

cylinder defined by its radius, material type core, and coating layer and its center coordinate with respect to the global cylindrical coordinate system $(\rho, \phi)$. In this analysis, $e^{+j \omega t}$, time dependence, has been used and suppressed throughout. 


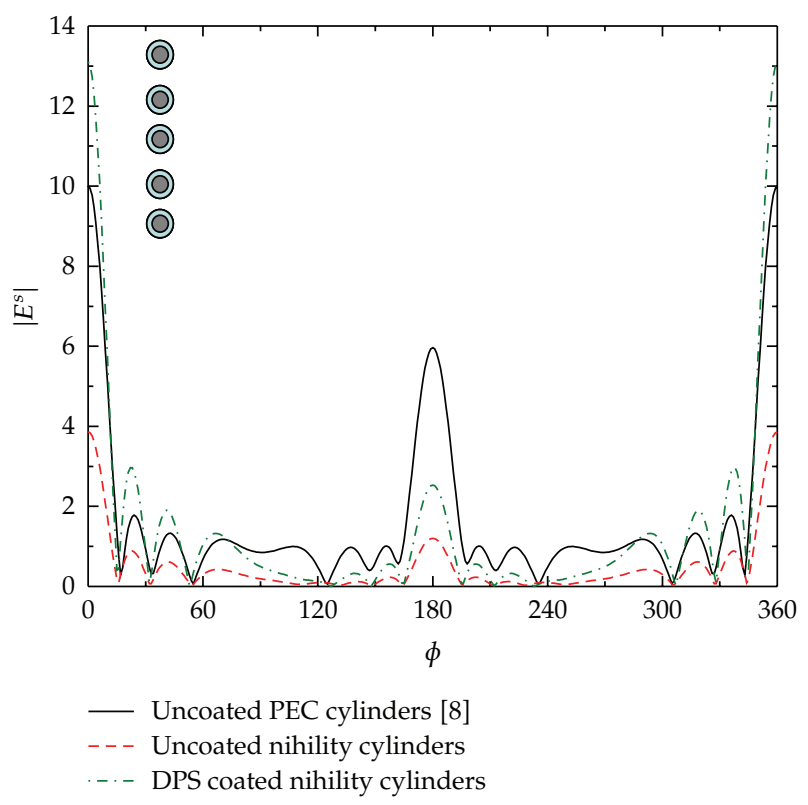

Figure 3: Far-zone scattered field pattern due to DPS $\left(\varepsilon_{1}=5, \mu_{1}=1\right)$ coated nihility circular cylinders (vertical configuration), $a=0.1 \lambda_{0}, b=0.12 \lambda_{0}$, and $d=0.75 \lambda_{0}$.

Consider an E-polarized incident plane wave to be the incident field on " $i$ th", cylinder is expressed in its local cylindrical coordinates $\left(\rho_{i}, \phi_{i}\right)$, as

$$
\begin{aligned}
E_{z}^{\mathrm{inc}}\left(\rho_{i}, \phi_{i}\right) & =e^{j k_{0} \rho_{i}^{\prime} \cos \left(\phi_{i}^{\prime}-\phi_{0}\right)} e^{j k_{0} \rho_{i} \cos \left(\phi_{i}-\phi_{0}\right)} \\
& =e^{j k_{0} \rho_{i}^{\prime} \cos \left(\phi_{i}^{\prime}-\phi_{0}\right)} \sum_{n=-\infty}^{\infty} j^{-n} J_{n}\left(k_{0} \rho_{i}\right) e^{j n\left(\phi_{i}-\phi_{0}\right)}
\end{aligned}
$$

where $\rho_{i}^{\prime}$ and $\phi_{i}^{\prime}$ represent the location of "ith" cylinder with respect to origin, $k_{0}$ is the free space wave number, $J_{n}(\cdot)$ is the Bessel function of first kind, and $\phi_{0}$ is the angle of incidence of the plane wave with respect to the $x$-axis. The corresponding $\phi$ component of the magnetic field is given by

$$
H_{\phi_{i}}^{\mathrm{inc}}\left(\rho_{i}, \phi_{i}\right)=\frac{1}{j \eta_{0}} e^{j k_{0} \rho_{i}^{\prime} \cos \left(\phi_{i}^{\prime}-\phi_{0}\right)} \sum_{n=-\infty}^{\infty} j^{-n} J_{n}^{\prime}\left(k_{0} \rho_{i}\right) e^{j n\left(\phi_{i}-\phi_{0}\right)} .
$$

Prime represents derivative with respect to the argument, and $\eta_{0}=\sqrt{\mu_{0} / \epsilon_{0}}$ is the impedanceof free space. The scattered fields by the "ith" cylinder in Region 0 , that is, free 


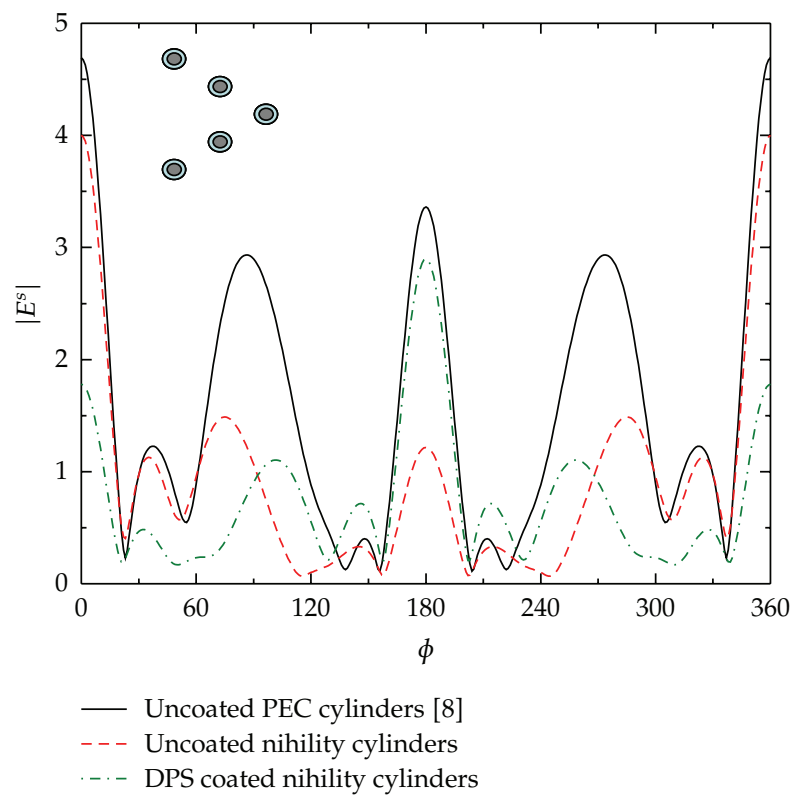

Figure 4: Far-zone scattered field pattern due to DPS $\left(\varepsilon_{1}=5, \mu_{1}=1\right)$ coated nihility circular cylinders (corner reflector configuration), $a=0.1 \lambda_{0}, b=0.12 \lambda_{0}$, and $d=0.75 \lambda_{0}$.

space, may be expressed as

$$
\begin{aligned}
E_{z i}^{0}\left(\rho_{i}, \phi_{i}\right) & =\sum_{n=-\infty}^{\infty} a_{i n} H_{n}^{(2)}\left(k_{0} \rho_{i}\right) e^{j n\left(\phi_{i}-\phi_{0}\right)}, \\
H_{\phi i}^{0}\left(\rho_{i}, \phi_{i}\right) & =\frac{1}{j \eta_{0}} \sum_{n=-\infty}^{\infty} a_{i n} H_{n}^{(2)^{\prime}}\left(k_{0} \rho_{i}\right) e^{j n\left(\phi_{i}-\phi_{0}\right)} .
\end{aligned}
$$

While the transmitted fields in region 1 , that is, inside the coating layer $\left(\eta_{1}=\sqrt{\mu_{1} / \epsilon_{1}}\right.$, $\left.k_{1}=\omega \sqrt{\mu_{1} \epsilon_{1}}\right)$ of the "ith" cylinder, may be written as

$$
\begin{aligned}
E_{z i}^{1}\left(\rho_{i}, \phi_{i}\right) & =\sum_{n=-\infty}^{\infty}\left[b_{i n} J_{n}\left(k_{1} \rho_{i}\right)+c_{i n} Y_{n}\left(k_{1} \rho_{i}\right)\right] e^{j n\left(\phi_{i}-\phi_{0}\right)}, \\
H_{\phi i}^{1}\left(\rho_{i}, \phi_{i}\right) & =\frac{1}{j \eta_{1}} \sum_{n=-\infty}^{\infty}\left[b_{i n} J_{n}^{\prime}\left(k_{1} \rho_{i}\right)+c_{i n} Y_{n}^{\prime}\left(k_{1} \rho_{i}\right)\right] e^{j n\left(\phi_{i}-\phi_{0}\right)},
\end{aligned}
$$

and the fields inside the core cylinder $\left(\eta_{2}=\sqrt{\mu_{2} / \epsilon_{2}}, k_{2}=\omega \sqrt{\mu_{2} \epsilon_{2}}\right)$ are given as

$$
\begin{aligned}
E_{z i}^{2}\left(\rho_{i}, \phi_{i}\right) & =\sum_{n=-\infty}^{\infty} d_{i n} J_{n}\left(k_{2} \rho_{i}\right) e^{j n\left(\phi_{i}-\phi_{0}\right)}, \\
H_{\phi i}^{2}\left(\rho_{i}, \phi_{i}\right) & =\frac{1}{j \eta_{2}} \sum_{n=-\infty}^{\infty} d_{i n} J_{n}^{\prime}\left(k_{2} \rho_{i}\right) e^{j n\left(\phi_{i}-\phi_{0}\right)},
\end{aligned}
$$




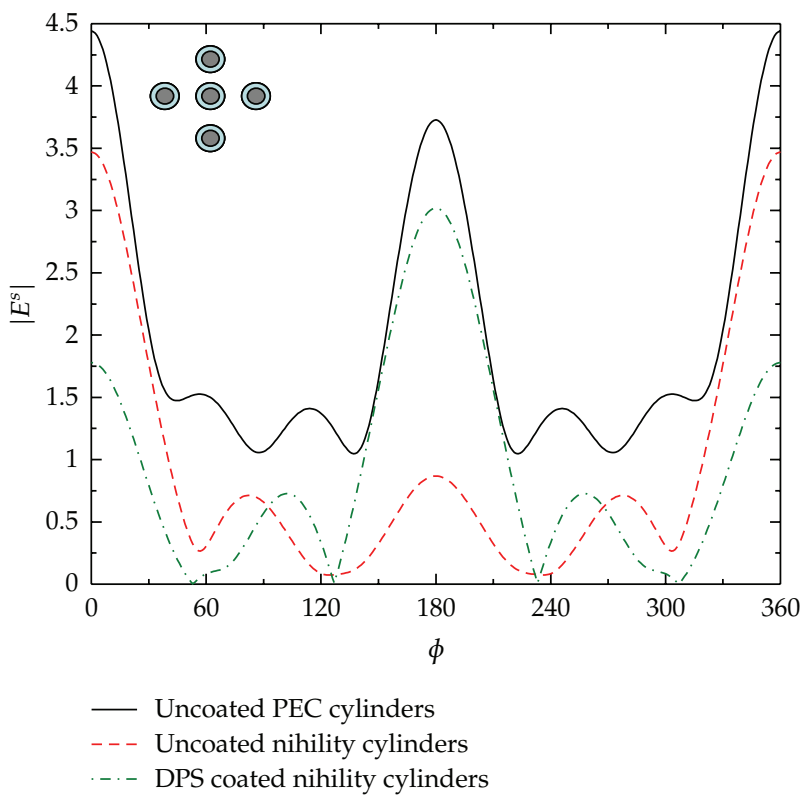

Figure 5: Far-zone scattered field pattern due to DPS $\left(\varepsilon_{1}=5, \mu_{1}=1\right)$ coated nihility circular cylinders (star configuration), $a=0.1 \lambda_{0}, b=0.12 \lambda_{0}$, and $d=0.75 \lambda_{0}$.

where $a_{i n}, b_{i n}, c_{i n}$, and $d_{i n}$ are the unknown scattering coefficients of the fields in different regions.

\section{Solution of the Unknown Scattering Coefficients}

The unknown scattering coefficients may be calculated by applying the boundary conditions at the surface of core and coating of the $i$ th cylinder. The continuity of the tangential components of electric and magnetic fields at the surface of the core, that is, $\rho=a_{i}$ of the $i$ th cylinder, is given as

$$
\begin{gathered}
E_{z i}^{1}=E_{z i}^{2} \quad \text { at } \rho_{i}=a_{i}, 0 \leq \phi_{i} \leq 2 \pi \\
H_{\phi i}^{1}=H_{\phi i}^{2} \text { at } \rho_{i}=a_{i}, 0 \leq \phi_{i} \leq 2 \pi .
\end{gathered}
$$

Using boundary conditions (3.1) and substituting the values of the field components, the unknowns may be expressed after some mathematical manipulation as

$$
b_{i l}=\left[\frac{\left(\eta_{2} / \eta_{1}\right) Y_{n}\left(k_{1} a_{i}\right) J_{n}^{\prime}\left(k_{2} a_{i}\right)-Y_{n}^{\prime}\left(k_{1} a_{i}\right) J_{n}\left(k_{2} a_{i}\right)}{J_{n}^{\prime}\left(k_{1} a_{i}\right) J_{n}\left(k_{2} a_{i}\right)-\left(\eta_{2} / \eta_{1}\right) J_{n}\left(k_{1} a_{i}\right) J_{n}^{\prime}\left(k_{2} a_{i}\right)}\right] c_{i l} .
$$




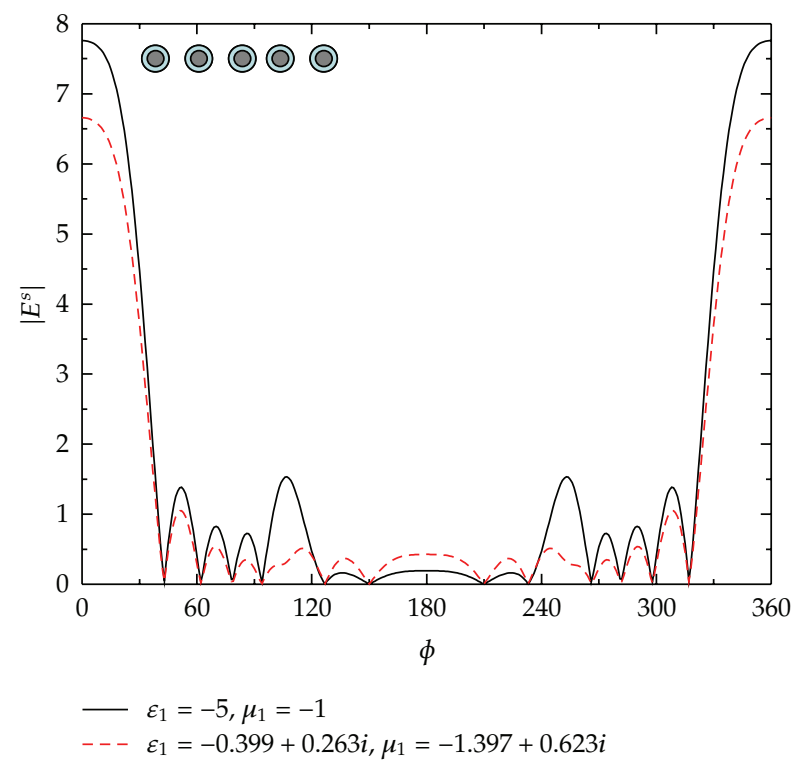

Figure 6: Far-zone scattered field pattern due to DNG coated nihility circular cylinders (horizontal configuration), $a=0.1 \lambda_{0}, b=0.12 \lambda_{0}$, and $d=0.75 \lambda_{0}$.

Now, the boundary conditions at the surface of coating layer of the $i$ th cylinder, that is, at $\rho=b_{i}$, are given as

$$
\begin{gathered}
E_{z i}^{\mathrm{inc}}+\sum_{g=1}^{M} E_{z i}^{0}=E_{z i}^{1} \quad \text { at } \rho_{i}=b_{i}, 0 \leq \phi_{i} \leq 2 \pi, \\
H_{\phi i}^{\mathrm{inc}}+\sum_{g=1}^{M} H_{\phi i}^{0}=H_{\phi i}^{1} \quad \text { at } \rho_{i}=b_{i}, 0 \leq \phi_{i} \leq 2 \pi .
\end{gathered}
$$

Since the fields given in equations (2.1)-(2.5) all are expressed in the local coordinates of the $i$ th cylinder, so addition theorem of Hankel functions is used to transfer these fields in terms of global coordinates. Generally, the transformation from $q$ th coordinates to the $p$ th coordinates is given for the $i$ th as [8]

$$
\begin{gathered}
H_{n}^{(2)}\left(k \rho_{q}\right) e^{j m \phi_{q}}=\sum_{m=-\infty}^{\infty} J_{m}\left(k \rho_{p}\right) H_{m-n}^{(2)}\left(k d_{p q}\right) e^{j m \phi_{p}} e^{-j(m-n) \phi_{p q}}, \\
d_{p q}^{2}=\rho_{p}^{\prime 2}+\rho_{q}^{\prime 2}-2 \rho_{p}^{\prime} \rho_{q}^{\prime} \cos \left(\phi_{p}^{\prime}-\phi_{q}^{\prime}\right), \\
\phi_{p q}= \begin{cases}\cos ^{-1}\left[\frac{\rho_{q}^{\prime} \cos \phi_{q}^{\prime}-\rho_{p}^{\prime} \cos \phi_{p}^{\prime}}{d_{p q}}\right], & \rho_{q}^{\prime} \sin \phi_{q}^{\prime} \geq \rho_{p}^{\prime} \sin \phi_{p}^{\prime}, \\
-\cos ^{-1}\left[\frac{\rho_{q}^{\prime} \cos \phi_{q}^{\prime}-\rho_{p}^{\prime} \cos \phi_{p}^{\prime}}{d_{p q}}\right], & \rho_{q}^{\prime} \sin \phi_{q}^{\prime}<\rho_{p}^{\prime} \sin \phi_{p}^{\prime},\end{cases}
\end{gathered}
$$




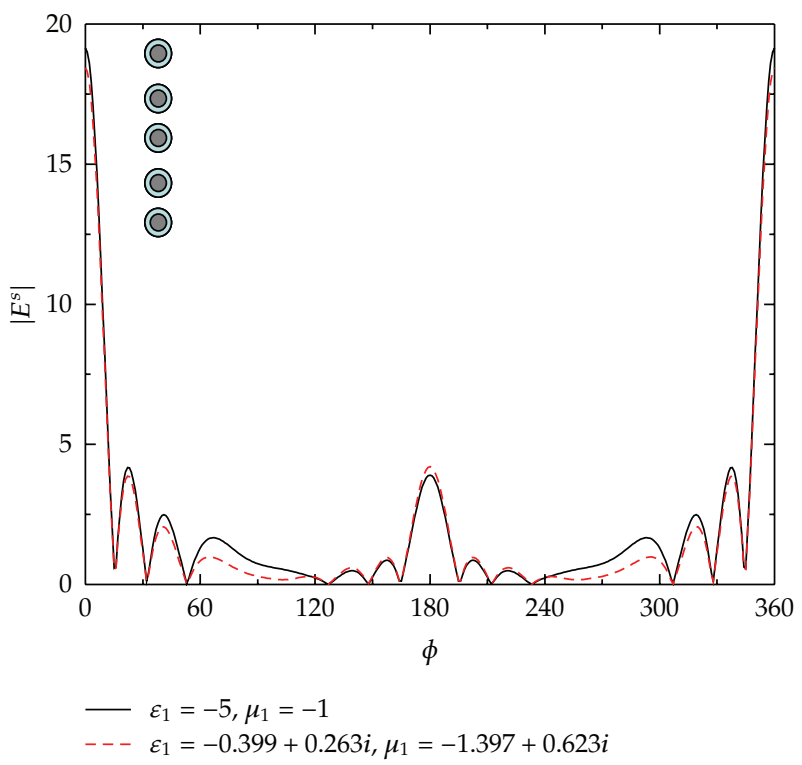

Figure 7: Far-zone scattered field pattern due to DNG coated nihility circular cylinders (vertical configuration), $a=0.1 \lambda_{0}, b=0.12 \lambda_{0}$, and $d=0.75 \lambda_{0}$.

where $\left(\rho_{q}^{\prime}, \phi_{q}^{\prime}\right)$ and $\left(\rho_{p}^{\prime}, \phi_{p}^{\prime}\right)$ are the origins of $q$ th and $p$ th coordinate systems, respectively. Solving the boundary conditions (3.3) for the unknowns $a_{i l}$ while applying the addition theorem of Hankel functions, we get

$$
\begin{aligned}
& V_{1 i}^{l}=\sum_{g=1}^{M} \sum_{m=-\infty}^{\infty} a_{g n} T_{1 i g^{\prime}}^{l n} \\
& V_{2 i}^{l}=\sum_{g=1}^{M} \sum_{m=-\infty}^{\infty} a_{g n} T_{2 i g^{\prime}}^{l n}
\end{aligned}
$$

where

$$
\begin{aligned}
& V_{1 i}^{l}=P_{l} J_{l}\left(k_{0} b_{i}\right), \\
& V_{2 i}^{l}=P_{l} J_{l}^{\prime}\left(k_{0} b_{i}\right), \\
T_{1 i g}^{l n}= & 0 \quad i=g, \quad l \neq n \\
= & H_{l}^{(2)}\left(k_{0} b_{i}\right) \quad i=g, \quad l=n \\
= & J_{l}\left(k_{0} b_{i}\right) H_{l n} \quad i \neq g, \\
T_{2 i g}^{l n}= & 0 \quad i=g, \quad l \neq n \\
= & H_{l}^{(2)}\left(k_{0} b_{i}\right) \quad i=g, \quad l=n \\
= & J_{l}^{\prime}\left(k_{0} b_{i}\right) H_{l n} \quad i \neq g,
\end{aligned}
$$




$$
\begin{gathered}
P_{l}=j^{l} e^{j k_{0} \rho_{i} \cos \left(\phi_{i}-\phi_{0}\right)}, \\
H_{l n}=H_{l-n}^{(2)}\left(k_{0} d_{i g}\right) e^{-j(l-n) \phi_{i g}} .
\end{gathered}
$$

In the above equations, the integers $l, n=0, \pm 1, \pm 2, \ldots, N_{i}$ and $i, g=0,1,2, \ldots, M$. All the summations range from minus infinity to plus infinity theoretically, but the upper limits $N_{i}$ are truncated to a numerical value which is related to the size and nature of the cylinders and also to the distance between the centers of the cylinders.

Equations (3.5) give the unknown scattering coefficients for the $i$ th cylinder. Following the same procedure on the rest of the cylinders, we obtain a matrix form solution as

$$
\left(\begin{array}{l}
V_{1} \\
V_{2}
\end{array}\right)=\left(\begin{array}{l}
T_{1} \\
T_{2}
\end{array}\right)(a) .
$$

Solving (3.7), we get the unknown scattering coefficients $a$ for all the cylinders. After truncating the infinite series solutions for the field expressions, solution of (3.7) gives the unknown scattering coefficients $a_{i n}$. Applying the limiting procedure proposed by Lakhtakia $[16,17]$, the scattered field from parallel nihility cylinders is obtained. The $H$-polarized incidence case can be deduced from E-polarized case using the duality theorem. The expressions of $H_{z}$ and $E_{\phi}$ fields in $H$-polarized case correspond to the $E_{z}$ and $H_{\phi}$ field expressions in the $E$-polarized incidence case.

\section{Simulations}

Far-field patterns from an array of five identical nihility cylinders of circular cross section, coated by different coating layers, are shown in this section. The radii of all the cylinders are taken the same as $a_{i}=0.1 \lambda_{0}$, while distance between their centers is taken to be $d$. The proposed geometries made by coated nihility cylinders are excited by plane wave propagating along positive $x$-axis. To check the validity of the formulation and numerical codes, scattered field patterns of the coated and uncoated parallel nihility cylinders have been compared with those obtained for the uncoated parallel perfect electric conducting (PEC) cylinders [8]. The code generated for the plots of the paper has been tested by comparison to plots for uncoated PEC cylinders [8].

Figures 2-5 present the far-field patterns due to different configurations of coated and uncoated nihility circular cylinders. Figure 2 shows the far-zone scattered field patterns of the five equally spaced coated nihility cylinders arranged along $x$-axis. The center-to-center distance between the cylinders in this arrangement is taken as $d=0.75 \lambda_{0}$. From this figure, it is seen that there is a big difference between the scattered field patterns of the uncoated nihility and PEC cylinders. And the back-scattered field due to nihility cylinders is smaller than that for the PEC cylinders. It is also observed from this figure that back-scattered field pattern due to double positive (DPS) $\left(\epsilon_{1}=5, \mu_{1}=1\right)$ coated nihility cylinders is comparable to that of uncoated PEC cylinders except that in this case forward and backward fields are almost equal. Figure 3 contains the far-field patterns of the five nihility cylinders placed along $y$-axis, with $d=0.75 \lambda_{0}$ as the spacing between their centers. This figure shows that in the 


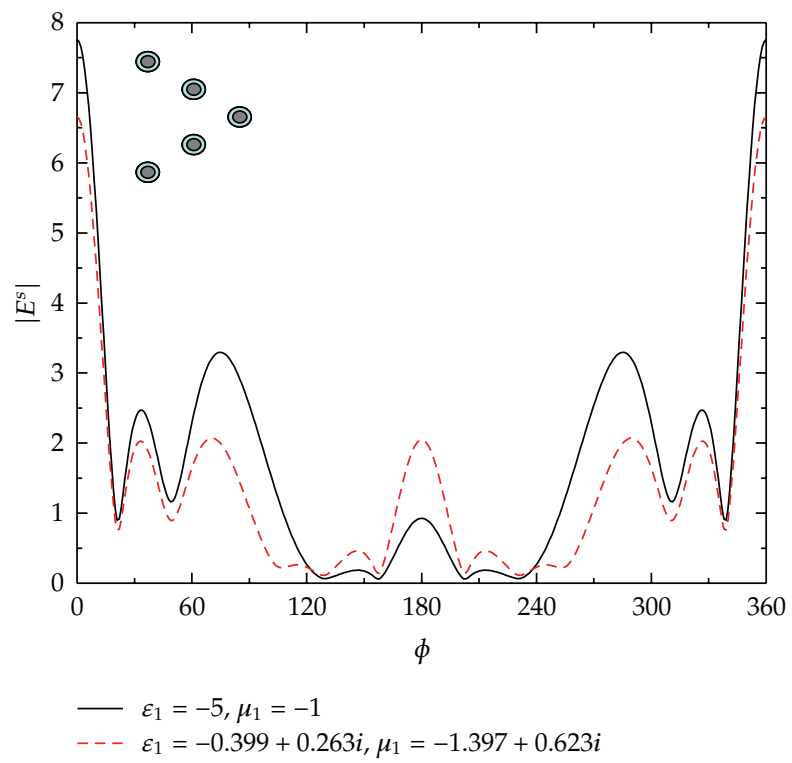

Figure 8: Far-zone scattered field pattern due to DNG coated nihility circular cylinders (corner reflector configuration) $a=0.1 \lambda_{0}, b=0.12 \lambda_{0}$, and $d=0.75 \lambda_{0}$.

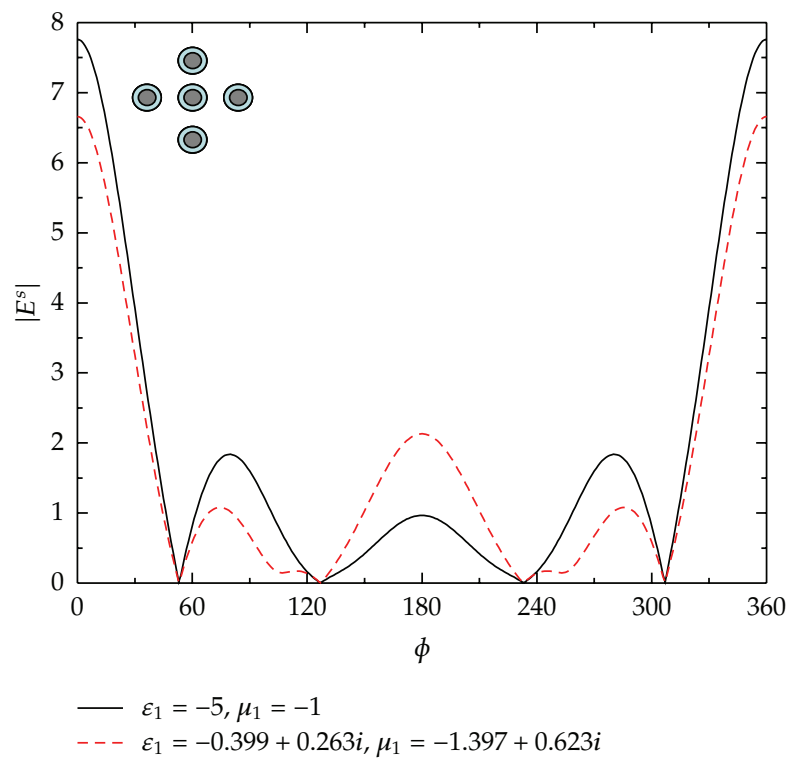

Figure 9: Far-zone scattered field pattern due to DNG coated nihility circular cylinders (star configuration), $a=0.1 \lambda_{0}, b=0.12 \lambda_{0}$, and $d=0.5 \lambda_{0}$.

case of DPS coated nihility cylinders, back-scattered field can be controlled when forwardscattered field increases.

Figure 4 contains the far-field patterns of the five nihility cylinders placed in corner reflector configuration, with $d=0.75 \lambda_{0}$ as the spacing between their centers. It is observed from the figure that in the case of DPS coated nihility cylinders, back-scattered field is greater 
than forward-scattered field in contrast to uncoated cases of nihility and PEC corner reflector configurations. Figure 5 presents the far-field patterns of the five nihility cylinders placed in a star-like configuration at the origin, with $d=0.5 \lambda_{0}$ as the spacing between their centers. In this case also, it is observed that in the case of DPS coated nihility cylinders, back-scattered field is greater than forward-scattered field in contrast to uncoated cases of nihility and PEC corner reflector configurations.

Figures 6-9 present the far-zone scattered field patterns of different arrangements of nihility circular cylinders coated by lossless double-negative (DNG) $\left(\epsilon_{1}=-5, \mu_{1}=-1\right)$ and dissipative and dispersive DNG $\left(\epsilon_{1}=-0.399+0.263 i, \mu_{1}=-1.397+0.623 i\right)$ materials. Figure 6 shows the reduction in forward and backward fields when coated by dissipative and dispersive DNG material. Figure 7 shows a very small variation due to lossless and dissipative and dispersive DNG materials. Figure 8 shows that in case of corner reflector formed by DNG coated nihility cylinders, forward-scattered field is reduced, while this backward-scattered field is increased for dissipative and dispersive DNG material. Figure 9 shows that in case of star-like configuration formed by DNG coated nihility cylinders, forward-scattered field is reduced, while the backward-scattered field is increased in case of dissipative and dispersive DNG material.

\section{Conclusions}

From the numerical results, it is observed that the forward-and backward-scattered fields can be altered or changed using different types of coating layers, on the nihility circular cylinders. Specially using DNG coating layers, a large contribution is obtained in the forward-scattered field as compared to the backward-scattered field.

\section{References}

[1] K. Hongo, "Multiple scattering by two conducting cylinders," IEEE Transactions on Antennas and Propagation, vol. 26, pp. 748-751, 1978.

[2] V. Twersky, "Multiple scattering of radiation by an arbitrary configuration of parallel cylinders," Journal of the Acoustical Society of America, vol. 24, pp. 42-46, 1952.

[3] J. E. Burke, D. Censor, and V. Twersky, "Exact inverse-separation series for multiple scattering in two dimensions," Journal of the Acoustical Society of America, vol. 37, pp. 5-13, 1965.

[4] H. A. Ragheb and M. Hamid, "Scattering by N parallel conducting circular cylinders," International Journal of Electronics, vol. 59, no. 4, pp. 407-421, 1985.

[5] H. A. Ragheb and M. Hamid, "Simulation of a cylindrical reflector by conducting circular cylinders," IEEE Transactions on Antennas and Propagation, vol. AP-35, no. 3, pp. 349-353, 1987.

[6] A. Z. Elsherbeni and M. Hamid, "Scattering by parallel conducting circular cylinders," IEEE Transactions on Antennas and Propagation, vol. AP-35, no. 3, pp. 355-358, 1987.

[7] A. Z. Elsherbeni and A. A. Kishk, "Modeling of cylindrical objects by circular dielectric and conducting cylinders," IEEE Transactions on Antennas and Propagation, vol. 40, no. 1, pp. 96-99, 1992.

[8] A. Z. Elsherbeni, M. Hamid, and G. Tian, "Iterative scattering of a gaussian beam by an array of circular conducting and dielectric cylinders," Journal of Electromagnetic Waves and Applications, vol. 7, no. 10, pp. 1323-1342, 1993.

[9] A. Z. Elsherbeni, "Comparative study of two-dimensional multiple scattering techniques," Radio Science, vol. 29, no. 4, pp. 1023-1033, 1994.

[10] W. Y. Yin and L. W. Li, "Multiple scattering from gyrotropic bianisotropic cylinders of arbitrary cross sections using the modeling technique," Physical Review E, vol. 60, no. 1, pp. 918-925, 1999.

[11] W. Y. Yin, L. W. Li, and M. S. Leong, "Scattering from multiple bi-anisotropic cylinders and their modeling of cylindrical objects of arbitrary cross-section," Progress in Electromagnetics Research, vol. 27, pp. 159-184, 2000. 
[12] B. H. Henin, A. Z. Elsherbeni, and M. Al Sharkawy, "Oblique incidence plane wave scattering from an array of circular dielectric cylinders," Progress in Electromagnetics Research, vol. 68, pp. 261-279, 2007.

[13] S. Ahmed and Q. A. Naqvi, "Electromagnetic scattering from parallel perfect electromagnetic conductor cylinders of circular cross-sections using an iterative procedure," Journal of Electromagnetic Waves and Applications, vol. 22, no. 7, pp. 987-1003, 2008.

[14] S. Ahmed and Q. A. Naqvi, "Electromagnetic scattering from a two dimensional perfect electromagnetic conductor (PEMC) strip and PEMC strip grating simulated by circular cylinders," Optics Communications, vol. 281, no. 17, pp. 4211-4218, 2008.

[15] J. A. Roumeliotis and A.-P. Ziotopoulos, "Electromagnetic scattering by a circular cylinder parallel with another one of small radius," Journal of Electromagnetic Waves and Applications, vol. 14, no. 1, pp. 27-43, 2000.

[16] A. Lakhtakia, "An electromagnetic trinity from "negative permittivity" and "negative permeability"," International Journal of Infrared and Millimeter Waves, vol. 22, no. 12, pp. 1731-1734, 2001.

[17] A. Lakhtakia, "An electromagnetic trinity from "negative permittivity" and "negative permeability", " International Journal of Infrared and Millimeter Waves, vol. 23, no. 6, pp. 813-818, 2002.

[18] A. Lakhtakia and J. B. Geddes III, "Scattering by a nihility cylinder," International Journal of Electronics and Communications, vol. 61, no. 1, pp. 62-65, 2007.

[19] A. Lakhtakia, M. W. McCall, and W. S. Weiglhofer, "Brief overview of recent developments on negative phase-velocity mediums (alias left-handed materials)," International Journal of Electronics and Communications, vol. 56, no. 6, pp. 407-410, 2002.

[20] S. A. Ramakrishna, "Physics of negative refractive index materials," Reports on Progress in Physics, vol. 68, no. 2, pp. 449-521, 2005.

[21] J. B. Pendry, "Negative refraction makes a perfect lens," Physical Review Letters, vol. 85, no. 18, pp. 3966-3969, 2000.

[22] A. Lakhtakia, "On perfect lenses and nihility," International Journal of Infrared and Millimeter Waves, vol. 23, no. 3, pp. 339-343, 2002.

[23] A. Lakhtakia and J. A. Sherwin, "Orthorhombic materials and perfect lenses," International Journal of Infrared and Millimeter Waves, vol. 24, no. 1, pp. 19-23, 2003.

[24] S. Ahmed and Q. A. Naqvi, "Scattering of electromagnetic waves by a coated nihility cylinder," Journal of Infrared, Millimeter, and Terahertz Waves, vol. 30, no. 10, pp. 1044-1052, 2009.

[25] Z. Shen and C. Li, "Electromagnetic scattering by a conducting cylinder coated with metamaterials," Progress In Electromagnetics Research, vol. 42, pp. 91-105, 2003.

[26] M. A. Mushref, "Transverse magnetic scattering of two incident plane waves by a dielectric coated cylindrical reflector," Central European Journal of Physics, vol. 3, no. 2, pp. 229-246, 2005.

[27] M. A. Mushref, "Closed solution to electromagnetic scattering of a plane wave by an eccentric cylinder coated with metamaterials," Optics Communications, vol. 270, no. 2, pp. 441-446, 2007.

[28] J. Sun, W. Sun, T. Jiang, and Y. Feng, "Directive electromagnetic radiation of a line source scattered by a conducting cylinder coated with left-handed metamaterial," Microwave and Optical Technology Letters, vol. 47, no. 3, pp. 274-279, 2005.

[29] E. Irci and V. B. Ertürk, "Achieving transparency and maximizing scattering with metamaterialcoated conducting cylinders," Physical Review E, vol. 76, no. 5, Article ID 056603, 2007. 


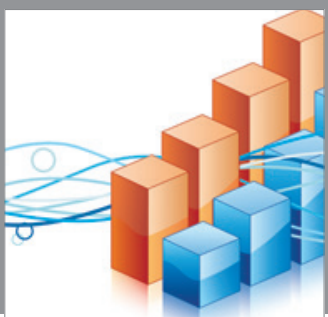

Advances in

Operations Research

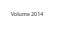

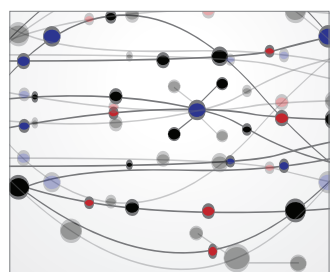

\section{The Scientific} World Journal
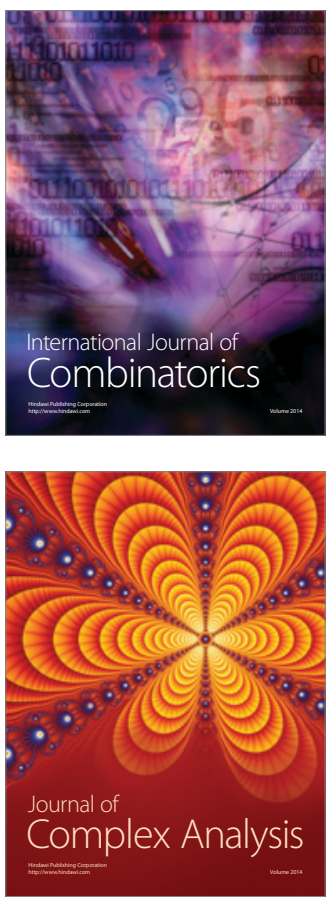

International Journal of

Mathematics and

Mathematical

Sciences
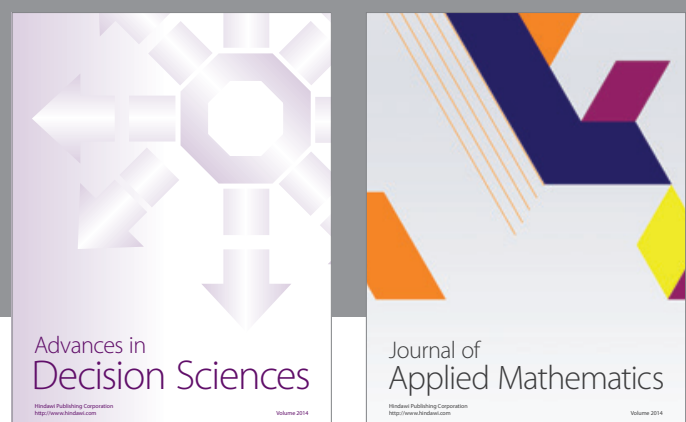

Journal of

Applied Mathematics
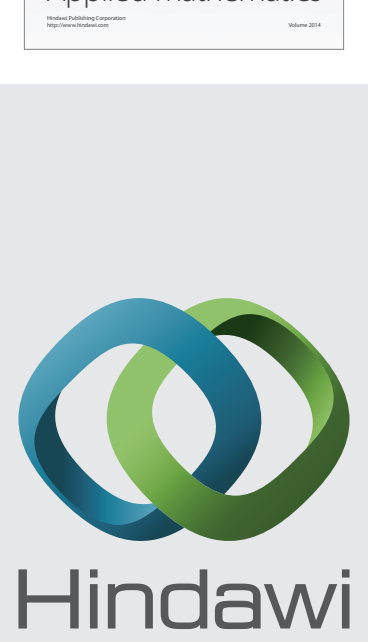

Submit your manuscripts at http://www.hindawi.com
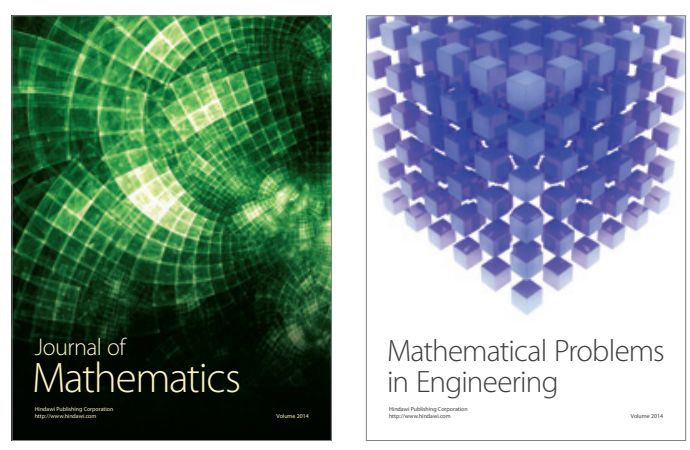

Mathematical Problems in Engineering
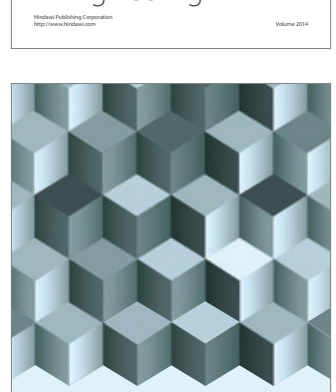

Journal of

Function Spaces
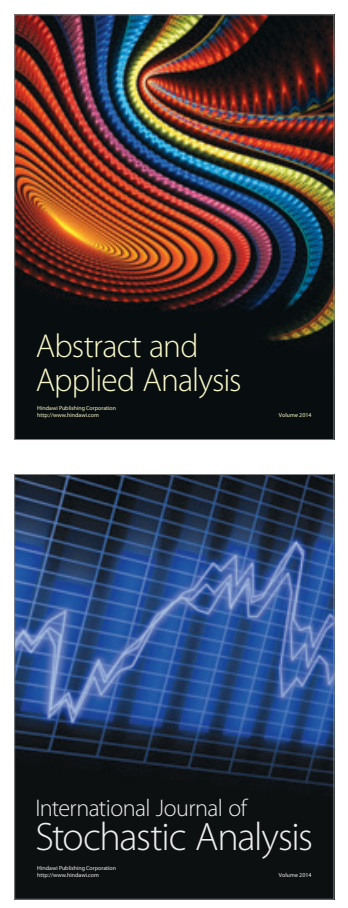

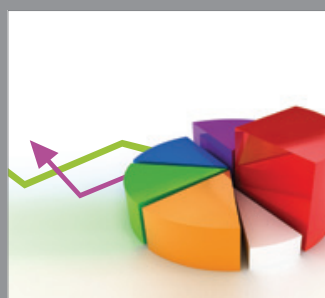

ournal of

Probability and Statistics

Promensencen
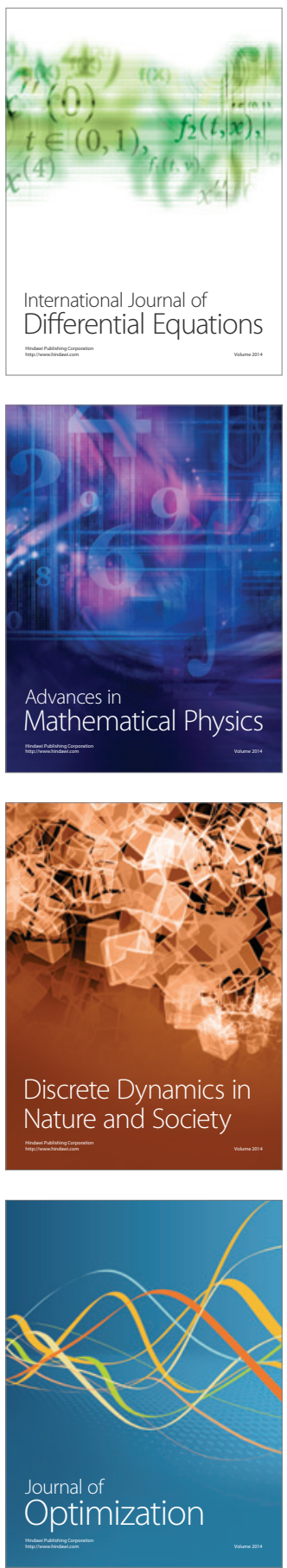